\title{
Characterization of Nanoscale Structures using a combination of an Ultra-High Resolution Field Emission SEM and Large Area Silicon Drift Detector.
}

\author{
N. Rowlands*, N. Erdman** and V. Robertson** \\ * Oxford Instruments, Suite 150, 300 Baker Avenue, Concord MA 01742 \\ ** JEOL, USA Inc., 11 Dearborn Road, Peabody MA 01960
}

\begin{abstract}
Although cold field emission scanning electron microscopes are well known for their ultra-high resolution capabilities at both low and high beam energy, it has generally been perceived that these FESEMs are not generally suited for all but the most routine analytical examination. Tradition $\mathrm{Si}(\mathrm{Li})$ or SDD detectors were developed with active areas between 10 and 30mm2. A new generation of SDD detectors is now available with active areas of up to $80 \mathrm{~mm} 2$. These detectors have the ability to collect and process in the order of hundreds of thousands of counts per second but also, due to large solid angles, can greatly increase the number of X-ray counts collected. This is especially important for samples that may be beam sensitive and need to be observed at lower $\mathrm{kVs}$ or where very small particles need to be identified in a bulk sample. A similar situation is encountered when STEM samples are presented for analysis in the SEM. In this case the increased peak to background ratios afforded by thin specimens are counteracted by the very small interaction volume.
\end{abstract}

This study demonstrated that a combination of the JEOL JSM 7500F SEM with an Oxford Instruments INCA system equipped with a 50mm2 X-Max detector provided adequate counts for EDS analysis in a wide variety of conditions. Samples were analyzed both on holey carbon films mounted on copper grids in STEM mode and on solid carbon substrates. X-ray maps and linescans were obtained where meaningful data could be acquired from the sample.

Catalytic Fe nanoparticles associated with carbon nanotubules (CNTs) were observed in bright field STEM mode at $30 \mathrm{kV}$ and $2.5 \mathrm{nA}$ at a working distance of $8 \mathrm{~mm}$. These were suspended on a holey carbon support overlying a $\mathrm{Cu}$ grid. Fe particles as small as $15 \mathrm{~nm}$ could be analyzed with peak to background values approaching that of a TEM.

Au nanoparticles on a carbon substrate were imaged at $8 \mathrm{kV}$ and 2.0nA using a low angle backscattered detector (LABE) and were analyzed in point mode. It can be observed in Figure 2 that $\mathrm{Au} \mathrm{M}$ lines were easily resolved on particles as small as $30 \mathrm{~nm}$ in less than 50 seconds.

Conclusions

A combination of cold field emission SEM and a 50mm2 SDD detector proved to be an excellent combination for the analysis of nanoparticles either on a solid substrate or in STEM mode. Ample counts were collected to easily characterize the particles in question and X-ray maps and linescans of fine structures were also obtained. 

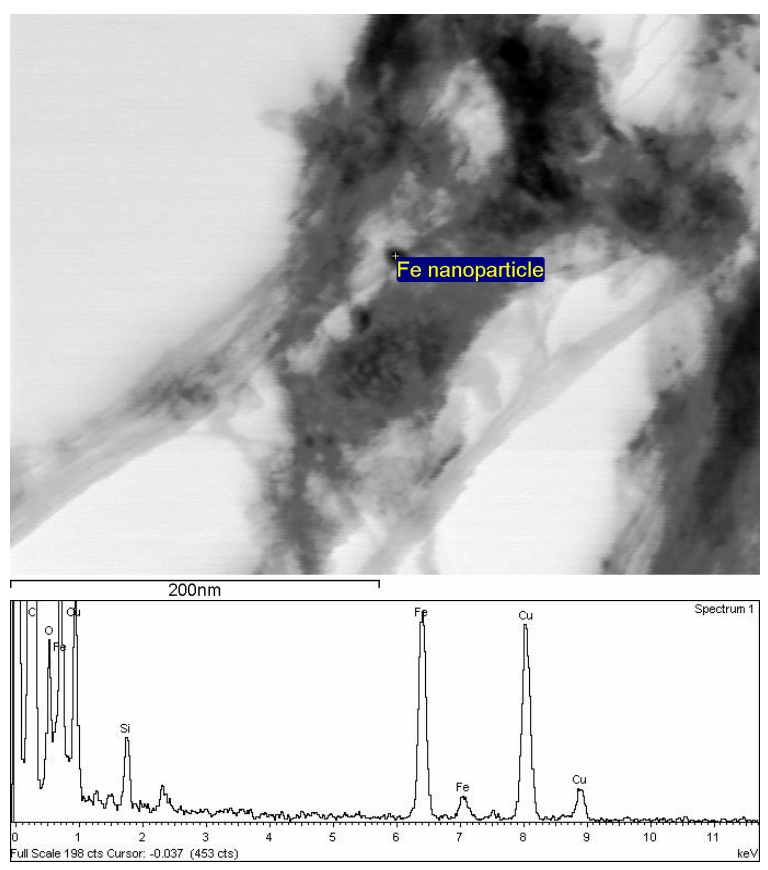

Figure1. Nano Fe particle STEM mode. Associated with CNTs on Cu Grid. 30kV 2.5nA 50secs. livetime.
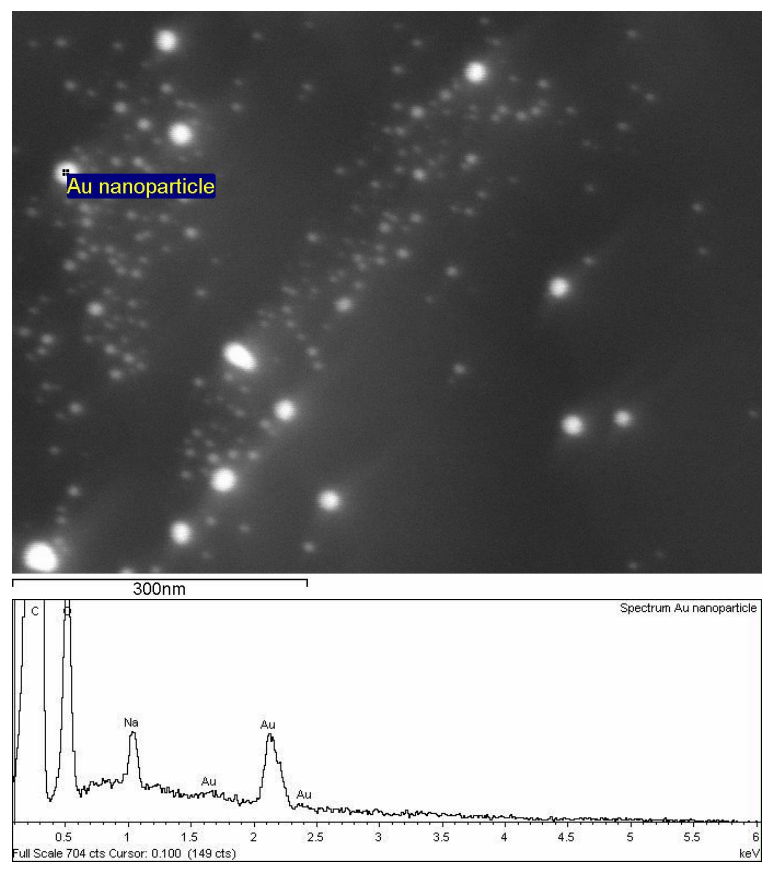

Figure 2. Au nanoparticles on solid $\mathrm{C}$ substrate. $8 \mathrm{kV}, 2.0 \mathrm{nA}, 20$ secs. livetime 